\title{
Impaired Mineral Metabolism in Cushing's Syndrome : Parathyroid Function, Vitamin D Metabolites and Osteopenia.
}

\author{
Nobuo KUGAI, Yoshinobu KOIDE*, Kamejiro YAMASHITA*, \\ TAKEHIDE SHIMAUCHI, NaOKazU NAGATA \\ AND OSAMU TAKATANI \\ The Third Department of Internal Medicine, National Defense Medical College, \\ Tokorozawa, Saitama 359 \\ *Institute of Clinical Medicine, University of Tsukuba, Sakura-mura, Ibaraki 305
}

\begin{abstract}
To clarify the mechanism for the impaired mineral metabolism in Cushing's syndrome, the clinical features, biochemical parameters before and after oral calcium load, and vitamin D metabolism were compared between two groups of patients of endogenous Cushing's syndrome (17 cases) with and without osteopenia. The patients with osteopenia [OP $(+): 7$ cases, all female] were older $(42.7 \pm 8.3$ y. o.) and had a longer duration $(117 \pm 75 \mathrm{M})$ of the syndrome than those without osteopenia [OP (-): $33.8 \pm 8.9$ y. o., $36 \pm 25 \mathrm{M}]$. OP $(-)$ showed a blunted hypercalciuria after oral calcium load $(63.7 \pm 20.4$ to $90.9 \pm 36.1 \mathrm{mg} / \mathrm{g} \cdot \mathrm{Cr})$, while OP $(+)$ had higher levels of urinary excretion of calcium (fasting: $120.4 \pm 37.5$, and after oral calcium load: $235.6 \pm$ $72.6 \mathrm{mg} / \mathrm{g} \cdot \mathrm{Cr})$, of cyclic AMP $(7.6 \pm 1.1 \mathrm{nmol} / \mathrm{dl} \cdot \mathrm{GF})$, and of plasma $1.25(\mathrm{OH})_{2} \mathrm{D}$ $(76.6 \pm 34.0 \mathrm{pg} / \mathrm{ml})$ than OP (-) (u-cAMP : $3.2 \pm 2.0 \mathrm{nmol} / \mathrm{dl} \cdot \mathrm{GF}, 1,25(\mathrm{OH})_{2} \mathrm{D}$ : $27.9 \pm 16.3 \mathrm{pg} / \mathrm{ml})$. These results indicate that 1 ) elderly female patients with Cushing's syndrome of long duration are susceptible to OP, 2) during the early phases of the syndrome, reduced intestinal calcium absorption with sustained calciuria (probably through the inhibition of calcium reabsorptive effect of PTH by glucocorticoid) induces negative calcium balance, leading to 3 ) a development of secondary hyperparathyroidism which stimulates $1,25(\mathrm{OH})_{2} \mathrm{D}$ synthesis. Thus, the mechanism involving bone resorption stimulated by excess PTH along with the direct inhibition of bone formation by glucocorticoid seems to play an important role in a progressive development of OP in Cushing's syndrome.
\end{abstract}

Patients with Cushing's syndrome suffer from an impairment of mineral metabolism, such as osteopenia and urolithiasis (Howland et al., 1958; Ross et al., 1966) as one of the major complications. Chronic administration of pharmacologic doses of glucocorticoid inhibits the intestinal calcium

Received November 22, 1985 absoption with normal or increased urinary excretion (Hahn et al., 1979; Klein et al., 1977 ; Lukert and Adams., 1976) leading to a persistent negative calcium balance which results in a progressive loss of bone mass (Hahn et al. 1976). However, the mechanisms, involved in these impairments of mineral homeostasis, especially in relation to the function of calcium regulating 
hormones, are still controversial. Some studies have demonstrated a secondary increase in parathyroid hormone (PTH) secretion (Findlings et al., 1982; Fucik et al., 1975; Hahn et al., 1979; Lukert and Adams., 1976; Suzuki et a1., 1983), while others have shown no difference in PTH secretion during hyper- and euglucocorticoid states (Seeman et al., 1980; Slovik et al., 1980). The previous reports on vitamin D metabolism indicated that glucocorticoids decrease (Klein et al., 1977; Lindgren et al., 1982 ; Suzuki et al., 1983), increase (Findlings et al., 1983; Hahn et al., 1981), or do not change (Hahn et al., 1977; Seeman et al., 1980 ; Slovik et al., 1980) plasma 25OHD or $1,25(\mathrm{OH})_{2} \mathrm{D}$. The heterogeneity of the subjects such as the presence or absence and clinical stage of underlying disease, dose and duration of glucocorticoid excess, and the degree of impairment of mineral metabolism might make some differences in these results.

To clarify the mechanism involved in the development of impaired mineral metabolism in Cushing's syndrome, a clinical study was carried out among the patients with endogenous Cushing's syndrome, and the clinical and biochemical features were compared between two groups of patients with and without osteopenia.

\section{Subject and Method}

\section{Subjects}

Seventeen patients with Cushing's syndrome (eight cases with Cushing's disease, eight cases with adrenal adenoma, and one case with ectopic ACTH syndrome, age range 18-52 y. o.) were studied. The diagnosis of Cushing's syndrome was established by the standard dexamethasone suppression test, adrenal scintigram, and plasma ACTH level.

Since the distribution of the osteopenia in Cushing's syndrome appears to involve predominantly the axial skeleton (vertebrae and rib) which is rich in trabecular bone (Hahn et al., 1974), the routine X-ray films of chest and abdomen were reviewed by radiologists for the evaluation of osteopenia (i. e. marked demineralization and vertebral compression fracture). According to the radiological reports, the patients were devided into two groups, one with marked changes of osteopenia and the other without such changes.

\section{Study Protocol}

After the patients were hospitalized and maintained on a diet which contains approximately $600 \mathrm{mg}$ of calcium and $10 \mathrm{~g}$ of sodium chrolide for at least 3 days, the basal plasma samples were obtained for the determination of immunoreactive PTH (iPTH) and vitamin D metabolites. The oral calcium load tests (Pak et al., 1975) were performed in ten patients.

\section{Analyses}

Serum iPTH was determined by a radioimmunoassay (RIA) kit (Eiken Immunochemical Laboratory, Ohji, Tokyo, Japan) (Koide et al., 1981). Vitamin D metabolites were measured by competitive protein binding assay [25OHD and $\left.24,25(\mathrm{OH})_{2} \mathrm{D}\right]$ or radioreceptor assay $\left[1,25(\mathrm{OH})_{2} \mathrm{D}\right]$ after the extraction of metabolites from plasma by Sephadex LH-20 and high pressure liquid chromatography (Imawari et al., 1982). Urinary cyclic AMP was determined by an RIA kit (Yamasa Shoyu, Chosi, Chiba, Japan). Serum and urinary calcium, phosphorus, and creatinine were measured by a multichannel autoanalyzer. The serum calcium level was corrected for protein binding (Payne et al., 1973). Statistical analyses were performed using Student's $t$ test.

\section{Results}

Clinical features (Table 1)

Seven patients, all female, showed marked changes of osteopenia. The age at the diagnosis of Cushing's syndrome was slightly older in patients with osteopenia than in those without it. The estimated duration with the clinical symptoms of the syndrome was significantly longer in patients with osteopenia than in those without it. The degree of hypercortisolism expressed by the serum cortisol level and urinary excretion of 17-OHCS was not different between two groups. 
Table 1. Clinical features of Cushing's syndrome in patients with and without marked osteopenia

\begin{tabular}{llccc}
\hline \hline Clinical features & & $\begin{array}{c}\text { OP }(+) \\
\mathrm{n}=7\end{array}$ & $\begin{array}{c}\text { OP }(-) \\
\mathrm{n}=10\end{array}$ & $P$ \\
\hline Sex & & $(\mathrm{M}: \mathrm{O}, \mathrm{F}: 7)$ & $(\mathrm{M}: 3, \mathrm{~F}: 7)$ & \\
Age & $(\mathrm{y} . \mathrm{o})$. & $42.7 \pm 8.3$ & $33.8 \pm 8.9$ & $<0.1$ \\
Duration & $(\mathrm{m})$ & $117 \pm 75$ & $36 \pm 25$ & $<0.01$ \\
Plasma cortisol & $(\mu \mathrm{g} / \mathrm{dl})$ & $22.8 \pm 5.8$ & $20.7 \pm 4.6$ & $\mathrm{NS}$ \\
Urinary 17-OHCS & $(\mathrm{mg} / \mathrm{g} \cdot \mathrm{Cr})$ & $16.2 \pm 5.6$ & $15.0 \pm 3.4$ & $\mathrm{NS}$ \\
\hline
\end{tabular}

OP: Osteopenia. Values are the mean \pm SD.

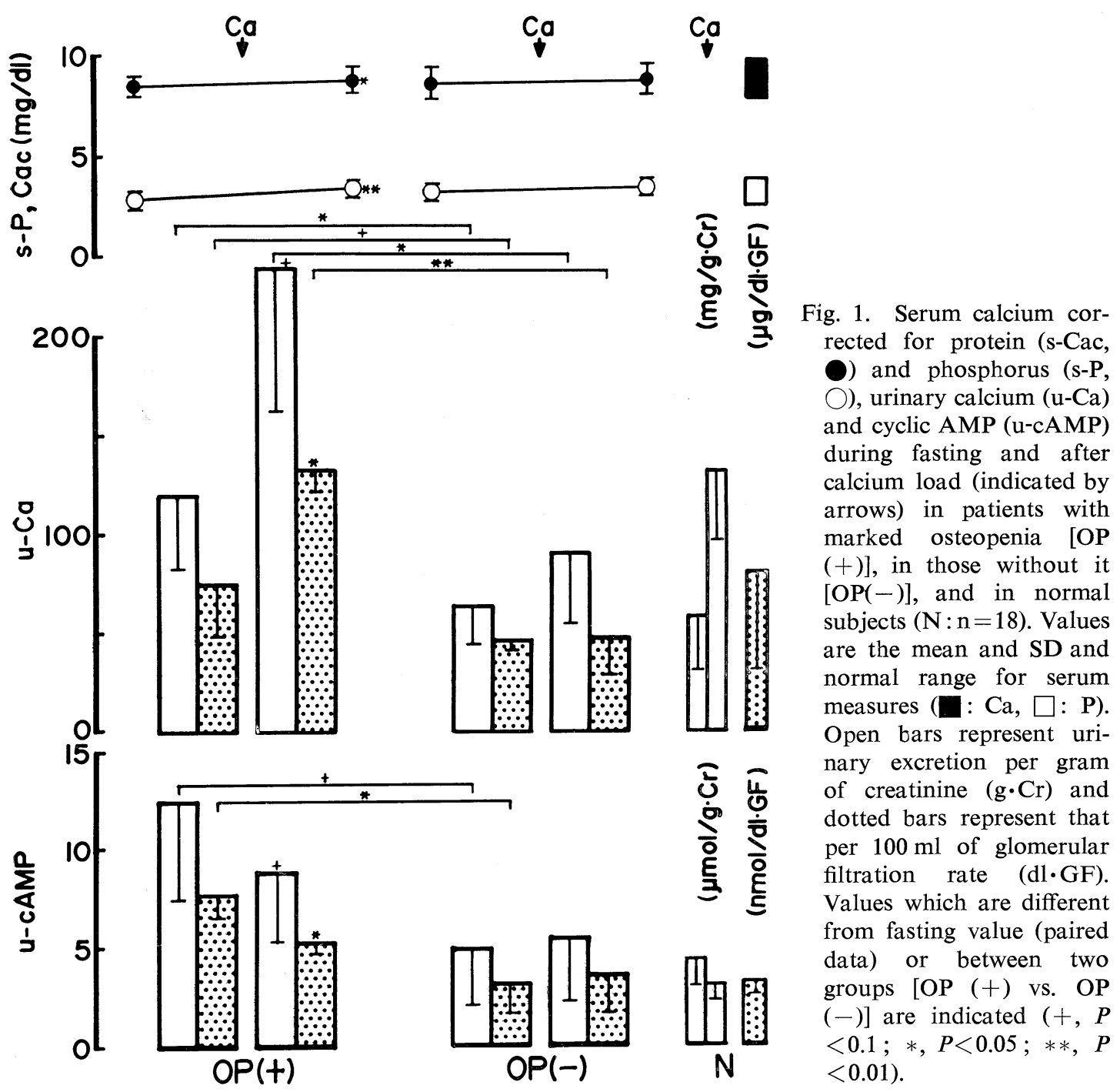




\section{Mineral metabolism}

Serum calcium corrected for protein and serum phosphorus were within the normal range and not different between two groups (Fig. 1, upper panels). In the patients without osteopenia, serum calcium and phosphorus did not change after oral calcium load. Patients with osteopenia showed a slight but significant increase in serum calcium and phosphorus levels after oral calcium load. In the patients without osteopenia, the mean urinary excretion of calcium during fasting was normal and did not increase significantly after oral calcium load (Fig, 1, right in the middle panels). In the patients with osteopenia, the mean urinary excretion of calcium during fasting was not in the hypercalciuric range but significantly higher than that of the patients without osteopenia. After oral calcium load, the mean urinary excretion of calcium increased by a degree compatible with that of normal subjects (Fig. 1, left in the middle panels).

\section{Parathyroid function}

Since only three cases with osteopenia had a detectable concentration of iPTH in plasma $(0.44 \pm 0.12 \mathrm{ng} / \mathrm{ml}$, detection limit of $0.2 \mathrm{ng} / \mathrm{ml}$ ), the parathyroid function was estimated from the urinary excretion of cyclic AMP. In the patients without osteopenia, the mean urinary excretion of cyclic AMP was normal and not suppressed after oral calcium load (Fig. 1, right in lower panels). In the patients with osteopenia, the mean urinary excretion of cyclic AMP was above the upper limit of the normal range and significantly higher than that of the patients without osteopenia. The mean urinary excretion of cyclic AMP was decreased significantly after oral calcium load in the patients with osteopenia (Fig. 1, left in the lower panels).

\section{Vitamin $D$ metabolites (Table 2)}

Plasma levels of $25 \mathrm{OHD}$ and $24,25(\mathrm{OH})_{2} \mathrm{D}$ were in the lower range of those for normal subjects, and the mean levels of these metabolites were not different between two groups.

The plasma levels of $1,25(\mathrm{OH})_{2} \mathrm{D}$ in the patients without osteopenia were in the lower range of those for normal snbjects and those in the patients with osteopenia were in the upper range of those for normal subjects. The mean plasma level of the metabolite was significantly higher in the patients with osteopenia than that in the patients without it.

\section{Discussion}

In the present study, the analyses of the clinical features revealed that the patients with Cushing's syndrome with marked osteopenia were all female and had a longer duration of the syndrome than those without osteopenia. The clinical study on the mineral metabolism demonstrated that the cases without osteopenia showed a

Table 2. Vitamin D metabolites in patients with and without marked osteopenia

\begin{tabular}{llcccc}
\hline \hline Metabolites & & Normal & $\begin{array}{c}\text { OP }(+) \\
\mathrm{n}=4\end{array}$ & $\begin{array}{c}\text { OP }(-) \\
\mathrm{n}=7\end{array}$ & $P$ \\
\hline $250 \mathrm{HD}$ & $(\mathrm{ng} / \mathrm{ml})$ & $19 \pm 5$ & $13.88 \pm 3.71$ & $16.29 \pm 4.75$ & $\mathrm{NS}$ \\
& & $(10-31)$ & $(9-18)$ & $(10-23)$ & \\
$24,25(\mathrm{OH})_{2} \mathrm{D}$ & $(\mathrm{ng} / \mathrm{ml})$ & $2.3 \pm 1.4$ & $0.30 \pm 0.28$ & $0.67 \pm 0.32$ & $\mathrm{NS}$ \\
& & $(0.4-4.8)$ & $(0.1-0.5)$ & $(0.2-1.1)$ & \\
$1,25(\mathrm{OH})_{2} \mathrm{D}$ & $(\mathrm{pg} / \mathrm{ml})$ & $37 \pm 11$ & $76.60 \pm 34.00$ & $27.90 \pm 16.31$ & $<0.01$ \\
& & $(26-62)$ & $(40-120.9)$ & $(7.5-49.5)$ & \\
\hline
\end{tabular}

OP: Osteopenia. Values are the mean $+\mathrm{SD}$ and range in parentheses. 
blunted hypercalciuric response after oral calcium load, while the cases with marked osteopenia had higher levels of urinary excretion of calcium (fasting and after oral calcium load), of cyclic AMP, and of plasma $1,25(\mathrm{OH})_{2} \mathrm{D}$ than those without osteopenia.

Since the patients with marked osteopenia were all female and older than those without osteopenia, the elderly female patients with Cushing's syndrome seem to be more susceptible to osteopenia. A similar finding has been reported in steroid induced osteopenia among patients with rheumatoid arthritis, and interpreted as a reflection of the reduced bone mass already present in elderly female patients before initiation of glucocorticoid administration (Saville and Kharmosh. 1967).

The long duration of Cushing's syndrome seems to play an important role in the development of osteopenia. Since the close correlation between the development of osteopenia and the duration of glucocorticoid administration has been demonstrated (Hahn et al., 1981), our findings indicate that in endogenous Cushing's syndrome, the duration of hypercortisolism also contributes to the development of osteopenia.

The blunted hypercalciuric response after oral calcium load seen in the cases without marked osteopenia reflects an impairment of intestinal calcium absorption as reported previously in glucocorticoid excess (Gallaher et al., 1973; Hahn et al 1979; Kimberg et al., 1971; Lukert et al., 1973). The mechanism involved in this impairement has been attributed to either the derangement of hepatic production of 25OHD (Avioli et al., $1968)$, the low level of $1,25(\mathrm{OH})_{2} \mathrm{D}$ in plasma (Lindgren et al., 1968) under glucocorticoid excess, or direct inhibition of calcium absorption by glucocorticoid under normal vitamin D metabolism (Kinberg et al., 1971; Seeman et al., 1980). In our patients without marked osteopenia who also showed impaired calcium absorption, the plasma levels of $25 \mathrm{OHD}$ and $1,25(\mathrm{OH})_{2} \mathrm{D}$ were distributed in the lower range of those for normal subjects. These findings seem to favor the mechanism of impaired calcium absorption involving abnormal vitamin D metabolism rather than the direct effects of glucocorticoid excess.

The impairment of calcium absorption induced by long term glucocorticoid would probably induce secondary hyperparathyroidism. Most studies have demonstrated an increase in PTH secretion during hypercortisolism (Findlings et al., 1982; Fucik et al., 1975; Hahn et al., 1979; Lukert and Adams. 1976; Suzuki et al., 1983), despite a few reports indicating no change in the plasma iPTH level during hyper- and euglucocorticoid states (Seeman et al., 1980 ; Slovik et al., 1980). In the present study, plasma iPTH concentrations were measurable only in a few cases with marked osteopenia. These controversy might be derived from the heterogeneity of the subjects, such as the degree of bone involvement or the duration of glucocorticoid excess. The methodological differences in RIA of PTH such as sensitivity and specificity with heterogeneous PTH fragments in plasma might also contribute to producing controversial results. Therefore, in the present study the parathyroid function was estimated using urinary excretion of cyclic AMP as an index. In the patients with marked osteopenia, the mean urinary excretion of cyclic AMP was elevated and suppressible by oral calcium load, suggesting the presence of secondary hyperparathyroidism. The elevation of serum phosphorus after oral calcium load probably reflects the suppression of phosphaturia induced by secondary hyperparathyroidism. While the excess PTH should increase tubular calcium reabsorption, the urinary excretion of calcium remained normal in patients with marked osteopenia. This finding may reflect the impaired 
calcium conservation by the kidney due to the interference with renal action of PTH by glucocorticold. Similar findings have demonstrated (Laake, 1960; Suzuki et al., 1983) and regarded as an important factor in the development of secondary hyperparathyroidism under glucocorticoid excess (Suzuki et al., 1983).

Regarding vitamin D metabolism in the hyperglucocorticoid state, plasma 25OHD or $1,25(\mathrm{OH})_{2} \mathrm{D}$ values have been reported to be decreased (Klein et al., 1977 ; Lindgren et al., 1982; Suzuki et al., 1983), increased (Findlings et al., 1983, Hahn et al., 1981), or unchanged (Hahn et al., 1977; Seeman et al., 1980; Slovik et al., 1980). In our series, plasma $25 \mathrm{OHD}$ and $24,25(\mathrm{OH})_{2} \mathrm{D}$ values were in the lower range of those for normal sujects either in the presence or in the absence of marked osteopenia. The mean plasma $1,25(\mathrm{OH})_{2} \mathrm{D}$ value for the entire subjects studied was within the range for normal subjects. However, the mean plasma $1,25(\mathrm{OH})_{2} \mathrm{D}$ level was significantly higher in the cases with marked osteopenia than in those without it, suggesting that glucocorticoid excess lasting long enough to cause osteopenia results in an increase in the plasma level of this metabolite. Since the plasma level of 25OHD was low and the serum phosphorus level was normal, the plasma $1,25(\mathrm{OH})_{2} \mathrm{D}$ level was elevated probably through parathyroid hormone excess.

The mechanism involved in these changes in parathyroid function and vitamin D metabolism are summarized as follows. During the early phase, hypercortisolism reduces intestinal calcium absorption probably by impairing vitamin $\mathrm{D}$ metabolism with sustained calciuria probably by inhibiting calcium reabsroption. This state of negative calcium balance for longer duration induces a secondary increase in PTH secretion to maintain a normal calcium balance. Excess PTH stimulates $1,25(\mathrm{OH})_{2} \mathrm{D}$ synthesis to normalize impaired intestinal calcium absorption. The urinary loss of calcium, however, continues leading to a persistent secondary hyperparathyroidism. Excess PTH also stimulates bone resorption resulting in osteopenia along with the direct inhibitory effects of glucocorticoid on bone formation (Jewsey and Riggs, 1970; Jee et al., 1970). A similarity in the distribution and histological changes of bone loss in glucocorticoid induced osteopenia and primary hyperparathyroidism (Hahn et al., 1974; Jee et al., 1980), and abolishment by parathyroidectomy of osteoclastic response to steroid administration in animals (Jee et al., 1970) indicate the importance of excess PTH in the development of the steroid induced osteopenia.

Although the single primary cause is difficult to define in advanced osteopenia in Cushing's syndrome, the functional states of calcium regulating hormones are somewhat different in the early stage from those in longstanding Cushing's syndrome. The understanding of these mechanisms will help in devising specific methods for treatment and prevention of the impairment of calcium metabolism under glucocorticoid excess based on the functional states of calcium regulating hormones.

\section{Acknowledgements}

We are indebted to Dr. Michio Imawari, Institute of Clinical Medicine, University of Tsukuba for the measurement of vitamin D metabolites.

\section{References}

Avioli, L. V., S. J. Birge and S. W. Lee (1968). Effects of prednisone on vitamin D metabolism in man. J. Clin. Endocrinol. Metab. 28, 13411346.

Caniggia, A., R. Nuti, F. Lore and A. Vittimo (1981). Pathophysiology of the adverse affects of glucoactive corticosteroids on calcium metabolism in man. J. Steroid Biochem. 15, 
153-161.

Findlings, J. W., N. D. Adams, J. Lemann, Jr., R. W. Gray, C. J. Thomas and J. B. Tyrrell (1982). Vitamin D metabolites and parathyroid hormone in Cushing's syndrome: relationship to calcium and phosphorus homeostasis. $J$. Clin. Endocrinol. Metab. 54, 1039-1044.

Fucik, R. F., S. C. Kukreja, G. K. Hargis, E. N. Bowser, W. J. Henderson and G. A. Williams (1975). Effects of glucocorticoids on function of the parathyroid glands in man. J. Clin. Endocrinol. Metab. 40, 152-155.

Gallagher, J. C., J. Aaron, A. Horsman, R. Wilkinson and B. E. Nordin (1973). Corticosteroid osteoporosis. Clin. Endoclinol. 2, 355-368.

Hahn, T. J., V. C. Boisseau and L. V. Avioli (1974). Effects of chronic corticosteroid administration on diaphyseal and metaphyseal bone mass. $J$. Clin. Endocrinol. Metab. 39, 274-282.

Hahn, T. J., L. R. Halstead and J. G. Haddad (1977). Serum 25-hydroxyvitamin D concentration in patients receiving chronic corticosteroid therapy. J. Lab. Clin. Med. 90, 399 $-404$.

Hahn, T. J., L. R. Halstead, S. L. Teitelbaum and B. H. Hahn (1979). Altered mineral metabolism in glucocorticoid induced osteopenia: effects of 25-hydroxy vitamin D administration. $J$. Clin. Invest. 64, 655-665.

Hahn, T. J., L. R. Halstead and D. T. Baran (1981). Effects of short term glucocorticoid administration on intestinal calcium absorption and circulating vitamin D metabolite concentrations in man. J. Clin. Endocrinol. Metab. 52, 111115.

Howland, W. J., D. G. Dugh and R. G. Sprague (1957). Roentgenologic changes in the skeletal system in Cushing's syndrome. Radiology. 71, 69-78.

Imawair, M., K. Kozawa, T. Yoshida and T. Osuga (1982). A simple and sensitive assay for 25-hydroxy vitamin D, 24,25-dihydroxy vitamin $\mathrm{D}$ and 1,25 -dihodroxy vitamin $\mathrm{D}$ in human serum. Clin. Chim. Acta 124, 63-73.

Jawsay, J. and B. L. Riggs (1970). Bone formation in hypercortisolism. Acta Endorcrinol. 63, 21-28.

Jee, W. S. S., H. Z. Park, W. E. Roberts and G. H. Kenner (1970). Corticosteroid and bone. Am. J. Anat. 129, 477-480.

Kimberg, D. V., R. D. Baerg, E. Gershon and R. T. Grauducius (1971). Effects of cortisone treatment on the active transport of calcium by the small intestine. J. Clin. Invest. 50, 1309 -1321 .

Klein, R. G., S. B. Arnaud, J. C. Gallagher, H. F. DeLuca and B. L. Riggs (1977). Intestinal calcium absorption in exogenous hypercortisonism: role of 25-hydroxy vitamin $\mathbf{D}$ and corticosteroid dose. J. Clin. Invest. 60, 253-259.

Koide, Y., N. Kugai, S. Kimura, T. Fujita, N. Yamashita, T. Hiramoto, J. Sukegawa, E. Ogata and K. Yamashita (1980). Increased 1,25dihydroxycholecalciferol as cause of abnormal calcium metabolism in sarcoidosis. J. Clin. Endocrinol. Metab. 52, 494-498.

Laake, H (1960). The action of corticosteroids on the renal absorption of calcium. Acta Endocrinol. 34, 60-64.

Lindgren, U., C. R. Merchant and H. F. DeLuca (1982). Effect of 1,25-dihydroxy vitamin $\mathbf{D}_{3}$ on osteopenia induced by prednisolone in adult rat. Calcif. Tissue Int. 34, 253-257.

Lukert, B. P., S. W. Stanbury and E. B. Mawer (1973). Vitamin D and intestinal transport of calcium: effects of prednisolone. Endocrinology 93, 718-722.

Lukert, B. P. and J. S. Adams (1976). Calcium and phosphorus homeostasis in man: effects of corticosteroids. Arch. Intern. Med. 136, 12491253.

Pak, C. Y. C., R. Kaplan, H. Bone, J. Townsend and O. Waters (1976). A simple test for the diagnosis of absorptive, resorptive and renal hypercalciurias. N. Engl. J. Med. 292, 497500.

Payne, R. B., A. J. Little, R. B. Williams and J. R. Milner (1973). Interpretation of serum calcium in patients with abnormal serum protein. Br. Med. J. 4, 643-646.

Ross, E. J., P. Marshall-Jones and M. Friedman (1966). Cushing's syndrome: diagnostic criteria. Q. J. Med. 25, 149-192.

Saville, P. D. and O. Kharmosh (1967). Osteoporosis of rheumatoid arthritis: influence of age, sex and corticosteroids. Arthritis Rheum. 10, 423-430.

Seeman, E., R. Kumar, G. G. Hunder, M. Scott, H. Heath, III and B. L. Riggs (1980). Production, degradation and circulating levels of 1,25-dihydroxy vitamin $\mathrm{D}$ in health and in chronic glucocorticoid excess. J. Clin. Invest. 66, 664-669.

Slovik, D. M., R. M. Ohman, F. C. Lowell, M. B. Clark, G. M. Segre and J. T. Potts, JR (1980). 
Parathyroid hormone and 25-hydroxy vitamin D levels in glucocorticoid treated patients. Clin. Endocrinol. 12, 243-248.

Suzuki, Y., Y. Ichikawa, E. Saito and M. Homma (1983). Importance of increased urinary calcium excretion in the development of secondary hyperparathyroidism of patients under glucocorticoid therapy. Metabolism 32, 151-156. 